\title{
A Rare Cause of Gastrointestinal Bleeding in a 65-Year-Old Man with History of Polycythemia Vera
}

\author{
Najmeh Aletaha 1, Hoda Hamid ${ }^{1, *}$, Niloofar Ayoobi Yazdi ${ }^{2}$, Reza Taslimi ${ }^{1}$, \\ Bijan Shahbazkhani ${ }^{1}$, Pardis Ketabi Moghadam ${ }^{3}$
}

1. Department of Gastroenterology, Tehran University of Medical Sciences, Imam Hospital, Tehran, Iran

2. Department of Radiology, Liver Transplantation Center, Tehran University of Medical Sciences, Tehran, Iran

3. Department of Internal Medicine, Shahid Beheshti Medical University, Tehran, Iran

\section{* Corresponding Author:}

Hoda Hamid, MD

Fellowship of Gastroenterology, Imam Khomeini hospital, Keshavarz boulevard, Tehran, Iran

Tel: +982166939010

Fax: +982166581615

Email: h-hamid@razi.tums.ac.ir

Received: 08 Jun. 2019

Accepted: 04 Sep. 2019

\section{ABSTRACT}

Polycythemia vera (PV) is classified as a myeloproliferative disorder (MPD). Such patients are prone to both thrombotic and hemorrhagic events. Although gastrointestinal (GI) bleeding is not a prominent manifestation of PV, it would be life threatening and necessitating hospital admission and blood transfusion if it occurs. GI hemorrhage in these patients may be due to Aspirin usage, peptic ulcer disease (PUD), acquired Von Willbrand disease, Dieulafoy lesion (DL), Mallory Weiss tear, and esophageal and gastric varices. DL is an enlarged, thick-walled artery in the muscularis mucosa with a small submucosal defect. In this case report, we describe a 65-year-old man with history of PV presented with a massive upper GI bleeding. After a therapeutic endoscopic hemostasis failure and reoccurrence of bleeding during hospital admission, an abdominal computed tomography $(\mathrm{CT})$ was ordered, which revealed an aberrant artery originated from aorta directly into the stomach. An angiographic embolization was considered for the patient, which was successfully performed. Our patient was complicated by splenic infarction due to splenic collateral arteries embolization and the overwhelming thrombotic tendency of the patient himself due to the history of PV. Fortunately, our patient's signs and symptoms responded to supportive therapies and eventually he discharged well.

\section{KEYWORDS:}

Gastrointestinal Bleeding, Polycythemia Vera, Angioembolization, Dieulafoy Lesion, Splenic Infarct

Please cite this paper as:

Aletaha N, Hamid H, Ayoobi Yazdi N, Taslimi R, Shabazkhani B, Ketabi Moghadam P. A Rare Cause of Gastrointestinal Bleeding in a 65-Year-Old Man with History of Polycythemia Vera. Middle East J Dig Dis 2019;11:225-229. doi:10.15171/mejdd.2019.153.

\section{INTRODUCTION}

Polycythemia vera (PV) is a chronic myeloproliferative disorder (MPD) in which stem cells overproliferate without any impairment in differentiation or maturation. Medical studies have reported a range of $12-40 \%$ for thrombotic events in patients with PV. It should also be considered that the incidence rate of hemorrhagic events in such patients is significant. Totally, reported PV complications are between $15 \%$ and $35 \% .{ }^{1}$ Gastrointestinal (GI) hemorrhages in these patients occur less frequently than other complications but would be overwhelming. It may be attributed to the use of Aspirin or anticoagulants, Von Willebrand disease, or concomitant thrombotic complications. For 
example, gastric or esophageal varices and their leading hemorrhages usually stem from abdominal vein thrombosis associated portal hypertention. ${ }^{2}$ In the present report, a 65 -year-old man with history of PV referred to Imam Hospital gastroenterology ward with recurrent upper GI bleeding is presented. Source of bleeding was not diagnosed by esophagogastroduodenoscopy and endoscopic treatment was not successful resulting in rebleeding. An aberrant artery originated from aorta directly into the stomach was diagnosed by computed tomography (CT), which was treated by angioembolization.

\section{CASE REPORT}

A 65-year-old man with history of PV (JAK2V617F mutation positive) since 40 years ago taking $80 \mathrm{mg}$ Aspirin daily and hydroxyurea 400 mg daily was admitted to the emergency ward of Imam Hospital with massive hematemesis without any previous abdominal discomfort. He was hemodynamically stable and no orthostatic change was detected at the time of his arrival. Physical examination revealed hepatosplenomegaly. The first complete blood counts, Prothrombin Time (PT), Partial Thromboplastin Time (PTT) and liver function tests were within normal limits. Isotonic intravenous fluid and intravenous proton pump inhibitor (PPI) were started and early endoscopy was performed. Endoscopy revealed that stomach was full of blood and clot. A large clot with oozing site was detected in the fundus, which had a submucosal prominence of about $1 \times 1.5 \mathrm{~cm}$. Epinephrine was injected in the periphery of hemorrhagic site and then Argon Plasma Coagulation (APC) was done. Second look endoscopy the day after admission showed an isolated fundal varix with a mass like prominence without active bleeding. Cyanoacrylate glue was injected. Abdominal sonography revealed hepatosplenomegaly and normal flow in portal, suprahepatic, and splenic veins without any thrombosis. On the fourth day of admission, our patient had another massive hematemesis which was apparently controlled by Sengstaken-Blakemore tube. But, hemoglobin $(\mathrm{Hb})$ level was plunged to $8.5 \mathrm{~g} / \mathrm{dL}$ in spite of packed cell transfusion and the next day examination revealed a blood leakage from Blakemore tube. A surgery consult and abdominal CT with and without IV contrast was requested, which revealed an aberrant artery originated from the celiac artery that was piercing into the fundus of stomach. Evidently, fundal oozing was attributed to a Dieulafoy lesion. Above that an old splenic arterial thrombosis and multiple collateral arteries around the pancreas were seen (figures 1, 2, 3, and 4). Portal and splenic veins were patent and were found without thrombosis. No evidence of left sided portal hypertension was detected in the angiogram (figure 5). The patient underwent celiac and mesenteric angiographic embolization with three vials of polyvinyl alcohol (PVA). There was no active bleeding from left gastric artery. Angiograms before and after selective embolization of the aberrant artery are depicted in figures 6,7 , and 8. At the $6^{\text {th }}$ day of admission, the patient started complaining of abdominal pain especially on the left upper quadrant. Another abdominal CT with IV contrast was done to evaluate probable mesenteric ischemia or gastric perforation. Imaging showed mild wall thickening of gastric fundus at the site of embolization with air bubbles in gastric fundus wall. No transmural necrosis or perforation was detected. Chronic splenic artery thrombosis and multiple peripancreatic collaterals were seen. Air bubbles were seen in small intraparenchymal vessels associated with some areas of intraparenchymal hemorrhage in favor of hemorrhagic infarct in medial aspect of the spleen (figure 9). Following the abdominal pain, the patient became febrile. Blood samples for cultures were sent and empiric antibiotic therapy was started. Symptomatic therapy was considered with analgesics. Daily abdominal sonography was done to find out if splenic abscess or rupture is forming or not. Sonography revealed two focal heteroechoic areas with the size of $80 \times 45$ $\mathrm{mm}$ and $85 \times 65 \mathrm{~mm}$ at the upper and lower poles of the spleen and a linear hyperechoic area in favor of air trapping caused by infarct. No collection or abscess formation was detected. Blood cultures were positive for Staph epidermidis. The course of the disease was finished after a 3-day trial of antibiotic therapy and the patient became afebrile and 


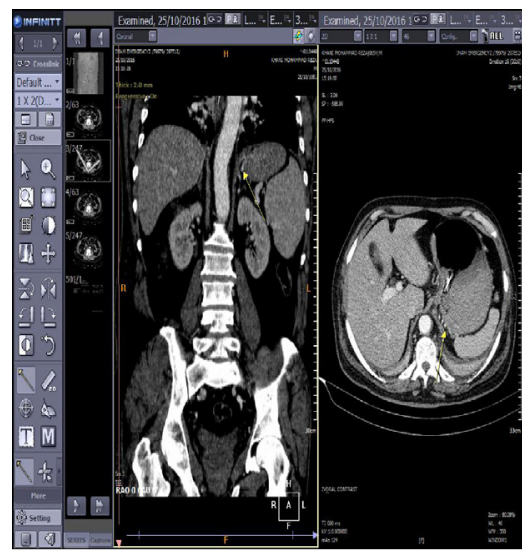

Fig.1: The origin at left side of celiac trunk (pointed by yellow arrow)

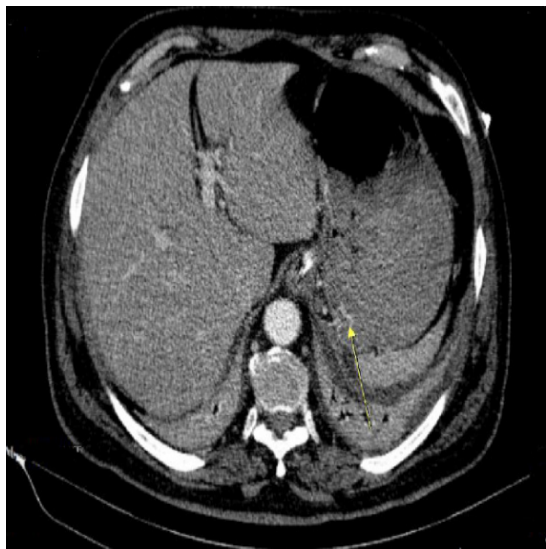

Fig.3, 4: Contrast extravasation (active bleeding pointed by yellow arrow)
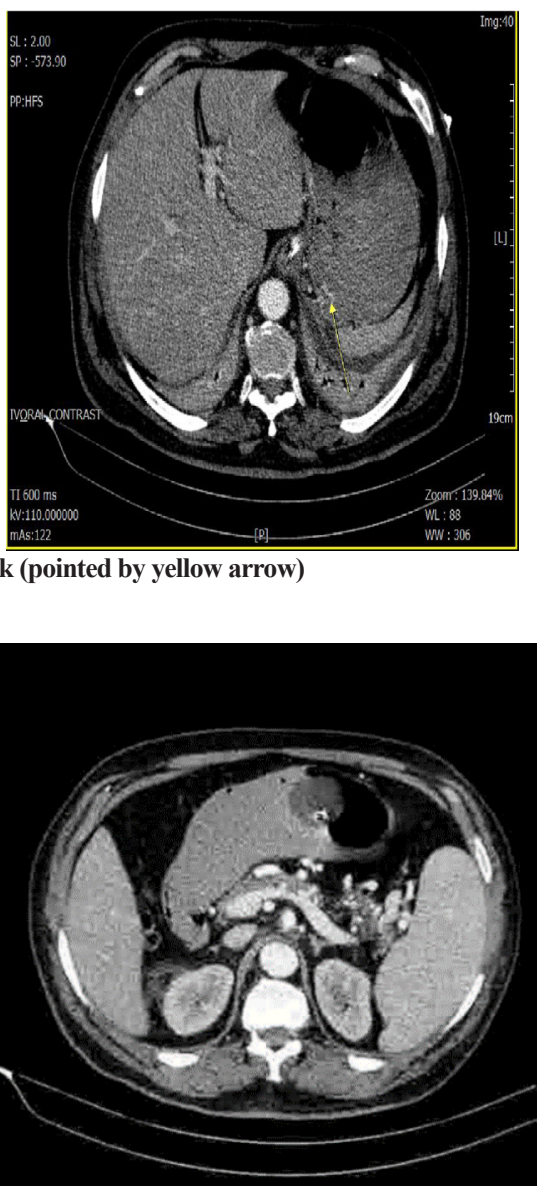

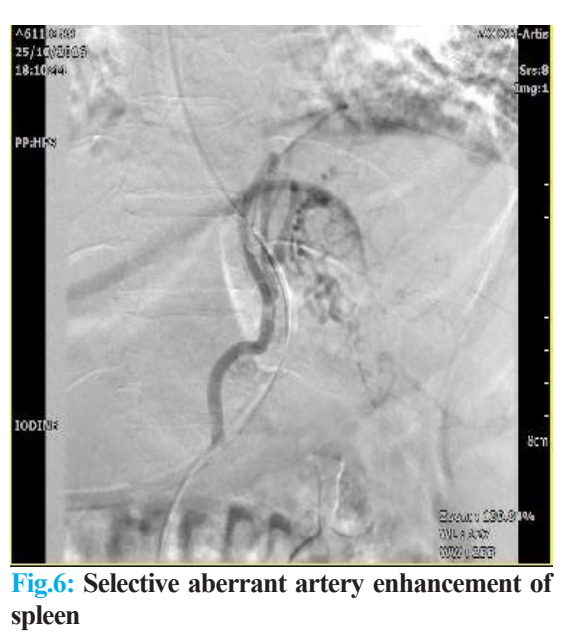

abdominal pain was gradually subsided. The course of antibiotic therapy was completed and finally the patient was discharged after 15 days of admission.

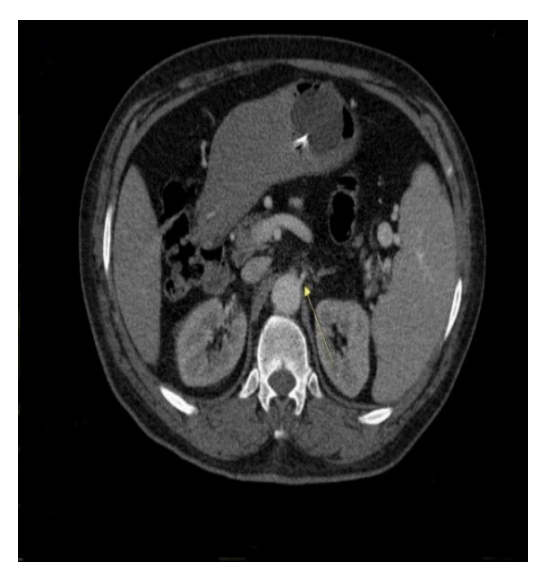

Fig.2: The origin at left side of celiac trunk (pointed by yellow arrow)

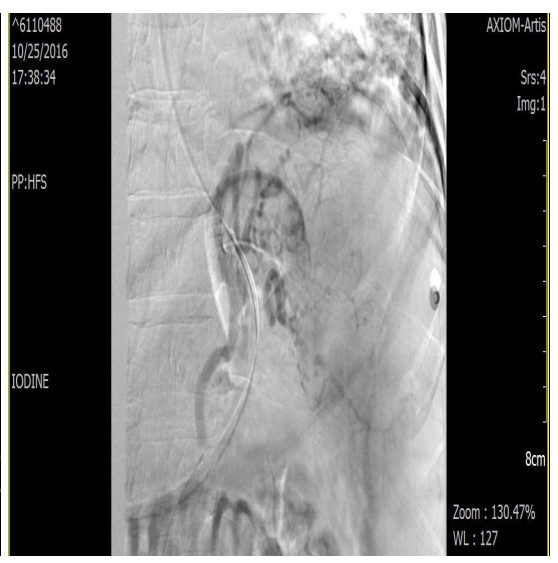

Fig.5: Portal and splenic veins are patent without thrombosis. No evidence of left sided portal hypertension was detected.

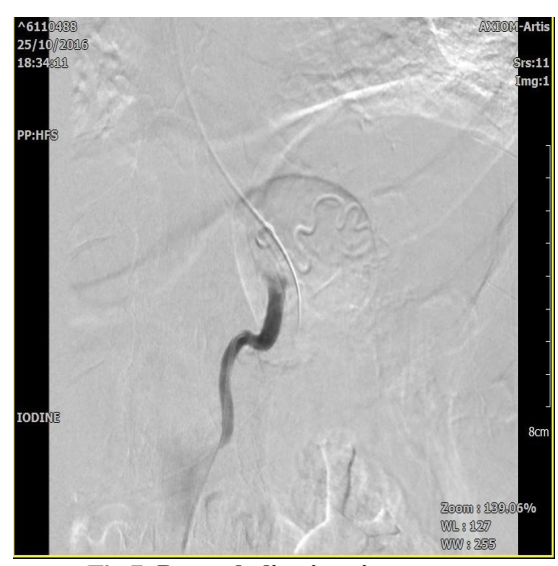

Fig.7: Pre embolization site

\section{DISCUSSION}

Dieulafoy lesion (DL) is a potentially rare and lifethreatening condition with high morbidity and mortality rate. About $1 \%$ to $2 \%$ of acute GI bleedings are attributed 


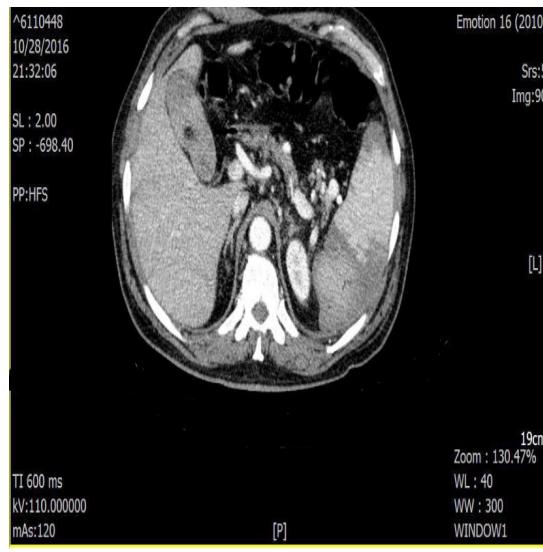

Fig.8: Post embolization angiogram

to this defect. Typically, these lesions are detected in the stomach, in particular in the proximal part of the lesser curvature although several cases with extragastric DLs in the esophagus, duodenum, jejunum, ileum, colon, rectum, appendix, and anal canal have been reported. Of these, the second and third most common locations are the duodenum and colon. ${ }^{3}$ DL was named after a French surgeon, Dieulafoy, who introduced three cases of upper GI bleeding in 1898 with similar reasons. The lesion was called by Dieulafoy as "exulceratio simplex", because of its tiny size and underlying large artery, which is tortuous but histologically normal. Although it was firstly understood that DL was an aneurysm of the vessels in the gastric wall but today it is accepted that it is a histologically confirmed large and tortuous sub-mucosal artery (a branch of the left gastric artery). Its protrusion and rupture into a tiny mucosal defect in particular in the proximal part of the stomach would culminate in a sudden massive hemorrhage. ${ }^{4}$ Pathological evaluation has ruled out the acquired, atherosclerotic, inflammatory, and aneurysmal nature of the lesion and has emphasized on the congenital basis of that. Reported rare cases of newborns with these lesions are in line with the accuracy of recent pathological investigations. ${ }^{5}$ Based on the data, any age-group could be affected but the elderly are more involved. These lesions are more common in men than women. Co-morbidities like cardiopulmonary disorders and chronic renal failure are present in $90 \%$ of patients. Usage of non-steroidal anti-inflammatory drugs (NSAIDs), Aspirin and warfarin has been detected in about half of the patients with DL. ${ }^{5}$ Esophagogastro-

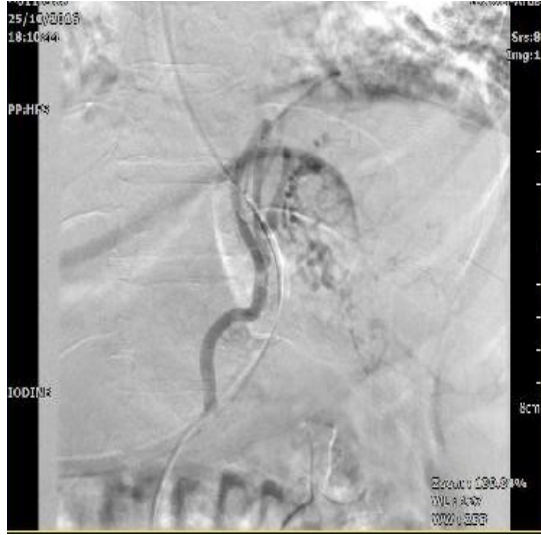

Fig.9: Post embolization splenic infarct

duodenoscopy has been successful in the diagnosis of DL in $70 \%$ of the gastric lesions. ${ }^{6}$ The fact that excessive blood and subtle characteristic of the lesion in $6 \%$ of the patients make endoscopic second or third look inevitable is well worth the usage of endoscopic ultrasound (EUS) for early detection of the lesions. ${ }^{5}$ DLs may be buried in mucosa and be presented as a protrusion mimicking gastric sub-mucosal tumors such as GI stromal tumors (GISTs). ${ }^{7}$ Use of angiography, CT angiography, and RBC scan has also been evidently accepted to localize the source of bleeding not being diagnosed by conventional endoscopic methods. ${ }^{5}$ Endoscopic therapies like thermal coagulation, regional injection, and mechanical banding have been accepted as the treatment of choice for easily accessible lesions with the success rate of $90 \%{ }^{3,5}$ There are some cases of silent DL without bleeding, which had been diagnosed after removal as a GIST by endoscopic sub-mucosal dissection (ESD). ${ }^{7}$ In spite of these procedures, DLs are still remain predictors of unfavorable outcome in non-variceal upper GI bleeding, ${ }^{4}$ following recurrent bleeding. ${ }^{8}$ Angiographic embolization has been successfully used for lesions not responding to endoscopic hemostatic methods. However, the risk of ischemia in these procedures should be taken into account. ${ }^{5}$ Currently, more and more patients become candidates for angioembolization because this procedure is minimally invasive and carries relatively low complications. ${ }^{4}$ The presented case illustrated an uncommon presentation of gastric sub-mucosal arterial bleeding from a DL originated from abdominal aorta instead of left gastric artery. The reason why this aberrancy was seen in this patient is not still clear whether it was attributed to PV and its characteristic complications like thrombosis 
and collateral arteries formation or it was a congenital variation. He was successfully treated by angioembolization. Our patient was complicated by splenic infarction, which was resolved after close follow-up and supportive care. Our patient was prone to severe bleeding during surgery because of multiple collateral arteries around the spleen, so surgery was held for plausible complications of splenic infarction like hemorrhage, rupture, abscess formation, or persistent pseudocyst. Fortunately, these complications were not detected in this case. An outpatient follow-up in 3 months showed no more complications in the patient.

\section{ETHICAL APPROVAL}

There is nothing to be declared.

\section{CONFLICT OF INTEREST}

The authors declare no conflict of interest related to this work.

\section{REFERENCES}

1. Yesilova AM, Yavuzer S, Yavuzer H, Cengiz M, Toprak ID, Hanedar E, et al. Analysis of thrombosis and bleeding complications in patients with polycythemia vera: a Turkish retrospective study. Int J Hematol 2016;105:70-78. doi: 10.1007/s12185-016-2105-0.

2. Elliott M A, Tefferi A. Thrombosis and haemorrhage in polycythaemia vera and essential thrombocythaemia. $\mathrm{Br}$ J Haematol 2005;128:275-90. doi: 10.1111/j.13652141.2004.05277.x.

3. Saleh R, Lucerna A, Espinosa J, Scali V. Case report: Dieulafoy lesion: the little known sleeping giant of gastrointestinal bleeds. Am J Emerg Med 2016;34: 2464.e3-2464.e5. doi: 10.1016/j.ajem.2016.06.024.

4. Scaramuzzi N, Winter D, Broe P, Lee MJ. Arterial embolization for Dieulafoy bleeding. Ir J Med Sci 2004;173:164-5. doi: 10.1007/BF03167934.

5. Baxter M, Aly EH. Dieulafoy's lesion: current trends in diagnosis and Management. Ann R Coll Surg Engl 2010;92:548-54. doi: 10.1308/003588410X1269966 3905311 .

6. Alomari AI, Fox V, Kaminb D, Afzal A, Arnold R, Chaudry G. Embolization of a bleeding Dieulafoy lesion of the duodenum in a child. Case report and review of the literature. J Pediatr Surg 2013;48:e3941. doi: 10.1016/j.jpedsurg.2012.10.055.

7. Chen X, Cao H, Wang S, Wang D, Xu M, Piao M, et al. Endoscopic submucosal dissection for silent gastric Dieulafoy lesions mimicking gastrointestinal stromal tumors: Report of 7 cases-a case report series. Medicine (Baltimore) 2016;95:e4829. doi: 10.1097/MD.0000000000004829.
8. Park SH, Lee DH, Park CH, Jeon J, Lee HJ, Lim SU, et al. Predictors of Rebleeding in Upper Gastrointestinal Dieulafoy Lesions. Clin Endosc 2015;48:385-91. doi: 10.5946/ce.2015.48.5.385. 